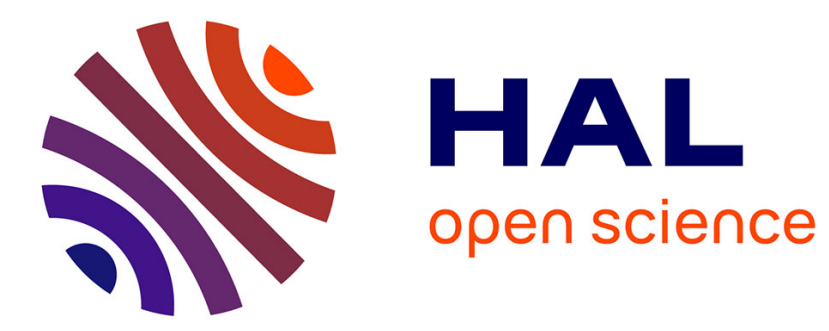

\title{
Yield-stress fluid deposition in circular channels
}

Benoit Laborie, Florence Rouyer, Dan E. Angelescu, Elise Lorenceau

\section{To cite this version:}

Benoit Laborie, Florence Rouyer, Dan E. Angelescu, Elise Lorenceau. Yield-stress fluid deposition in circular channels. Journal of Fluid Mechanics, 2017, 818 (2), pp.838 - 851. 10.1017/jfm.2017.161. hal-01611665

\section{HAL Id: hal-01611665 \\ https://hal.science/hal-01611665}

Submitted on 6 Oct 2017

HAL is a multi-disciplinary open access archive for the deposit and dissemination of scientific research documents, whether they are published or not. The documents may come from teaching and research institutions in France or abroad, or from public or private research centers.
L'archive ouverte pluridisciplinaire HAL, est destinée au dépôt et à la diffusion de documents scientifiques de niveau recherche, publiés ou non, émanant des établissements d'enseignement et de recherche français ou étrangers, des laboratoires publics ou privés. 


\title{
Yield-stress fluid deposition in circular channels
}

\author{
Benoît Laborie $^{1,3} \dagger$, Florence Rouyer ${ }^{2}$, Dan E. Angelescu ${ }^{3,4}$, and Elise \\ Lorenceau $^{1}$ \\ ${ }^{1}$ Université Paris-Est, Laboratoire Navier, UMR 8205 CNRS, ENPC ParisTech, IFSTTAR, 2 \\ allé Kepler, 77420 Champs-Sur-Marne, France \\ ${ }^{2}$ Université Paris-Est, Laboratoire Navier, UMR 8205 CNRS, ENPC ParisTech, IFSTTAR, 77 \\ 454 Champs-Sur-Marne, France \\ ${ }^{3}$ Université Paris-Est, ESIEE Paris/ESYCOM, 2 Bd. Blaise Pascal, 93162 Noisy le Grand, \\ France \\ ${ }^{4}$ Fluidion SAS, 231 Rue St. Honoré, 75001 Paris, France
}

(Received xx; revised xx; accepted xx)

Since the pioneering works of Taylor and Bretherton, the thickness $h$ of the film deposited behind a long bubble invading a Newtonian fluid is known to increase with the Capillary number power $2 / 3\left(h \sim R C a^{2 / 3}\right)$, where $R$ is the radius of the circular tube and the Capillary number, $C a$, comparing the viscous and capillary effects. This law, known as Bretherton law, is only valid in the limit of $C a<0,01$ and negligible inertia and gravity. We revisit this classical problem when the fluid is a Yield-Stress Fluid (YSF) exhibiting both a yield stress and a shear-thinning behaviour. First, we provide quantitative measurement of the thickness of the deposited layer for Carbopol HerschelBulkley fluid in the limit where the yield-stress is of similar order of magnitude as the capillary pressure and for $0.1<C a<1$. To understand our observation, we use scaling arguments to extend the analytical expression of Bretherton's law to YSF in circular tubes. In the limit of $C a<0,1$, our scaling law, in which the adjustable parameters are set using previous results concerning non-Newtonian fluid, successfully retrieves several features of the literature. First, it shows that $(i)$ the thickness deposited behind a Bingham YSF (exhibiting a yield stress only) is larger than for a Newtonian fluid and $(i i)$ the deposited layer increases with the amplitude of the yield stress. This is in quantitative agreement with previous numerical results concerning Bingham fluid. It also agrees with results concerning pure shear-thinning fluids in the absence of yield stress : the shear-thinning behaviour of the fluid reduces the deposited thickness as previously observed. Last, in the limit of vanishing velocity, our scaling law predicts that the thickness of deposited YSF converges towards a finite value, which presumably depends on the microstructure of the YSF, in agreement with previous research on the topic performed in different geometries. For $0.1<C a<1$, the scaling law fails to describe the data. In this limit, non-linear effects must be taken into account.

\section{Introduction}

When a plate or a fibre is drawn out of a liquid bath, a thin liquid film remains on its surface. Similarly, when a long bubble moves into a tube of radius $R$ which is pre-filled with a fluid, a thin layer of liquid remains on the wall of the channel as the bubble displaces the fluid. Finding how $h$, the thickness of the deposited film, varies with the

$\dagger$ Email address for correspondence: blaborie@lps.ens.fr 
different parameters driving the deposition of the fluid on solid walls has been extensively studied for various geometries and fluids, and is commonly referred to as "the coating or deposition problem".

The deposition of Newtonian fluids, in conditions where gravity and inertia are negligible compared to surface tension and viscous effects, was first investigated in the 1950's by Landau \& Levich (1942); Taylor (1961); Bretherton (1961); Cox (1962), they showed that $h$, the liquid layer deposited in a tube of radius $R$ scales as:

$$
\frac{h}{R} \sim C a^{\frac{2}{3}}
$$

Where $C a=\frac{\eta V}{T}$ is the capillary number, $V$ the bubble velocity, $\eta$ the fluid viscosity and $T$ the liquid air surface tension. Equation 1.1 is the classical Bretherton's law which compares remarkably well with experiments for $C a \ll 1$ (or, equivalently: $\frac{h}{R} \ll$ 1) (Bretherton 1961; Taylor 1961; Aussillous \& Quéré 2000), with a proportionality coefficient of 1.34, which depends on the length of the bubble as shown by Schwartz et al. (1986). For $C a \approx 1, h$ tends to a finite value $\left(\frac{h}{R} \simeq 0.4\right)$ as shown by Taylor (1961), and later by Cox (1962) and Reinelt \& Saffman (1985).

The deposition of non-Newtonian fluids, initially motivated by the development of photographic industry (Deryagin \& Levi 1964), has been widely used in various industrial processes to enhance, modify, or decorate the surface properties of chosen substrates. Indeed, coating materials, such as paints and lacquers commonly used to prevent corrosion in automotive industry, often behave like non-Newtonian fluids. The deposition of various non-Newtonian fluids, such as polymer solutions or elastic fluids, has been addressed theoretically, numerically or experimentally (Gutfinger \& Tallmadge 1965; Huzyak \& Koelling 1997; de Ryck \& Quéré 1998; Quéré 1999; Kamisli \& Ryan 1999; Gauri \& Koelling 1999; Kamisli \& Ryan 2001; Kamışlı 2003; Weinstein \& Ruschak 2004; Duggal \& Pasquali 2004; Behr et al. 2005; Quintella et al. 2007; Ashmore et al. 2008; Boehm et al. 2011). In particular, for a shear-thinning or shear-thickening fluid, with a stress/strain-rate relationship in simple shear given by $\tau=k \dot{\gamma}^{n}$ (where $n$ denotes the power-law index and $k$ the consistency (in Pa.s $\left.{ }^{n}\right)$ ), the same scaling arguments as for Newtonian fluids can be used (Gutfinger \& Tallmadge 1965; de Ryck \& Quéré 1998; Hewson et al. 2009), thus yielding:

$$
\frac{h}{R} \sim \widehat{C a}^{\frac{2}{2 n+1}}
$$

where $\widehat{C a}=\frac{k\left(\frac{V}{R}\right)^{n}}{T / R}$ is a modified capillary number for shear thinning/thickening fluids, which reduces to $C a$ for $n=1$.

The deposition of Yield-Stress fluids (YSF) has been investigated in different geometries. Such fluids are characterized by an intermediate fluid or solid behavior: they exhibit an elastic response below, and liquid flow response above a critical yield stress $\tau_{y}$. In the geometry of a plate withdrawn from a bath full of YSF, the thickness of the deposited layer was shown to be almost independent of the velocity but proportional to the material yield stress (Maillard et al. 2014; Boujlel \& Coussot 2013). Using particle imaging velocimetry, the authors observe that a uniform boundary layer builds up along the plate before emersion out of the bath. This boundary layer, set by a balance between the yield and gravity stresses also determines $h$, the thickness of the deposited layer, so that $h \propto \tau_{y}$, in good agreement with experimental data. This experimental result differs from earlier theoretical works concerning the deposition of purely plastic materials on a fibre, which predicts that $h \propto \tau_{y}^{2}$ (Deryagin \& Levi 1964; Quéré 1999). This discrepancy is 
due to the geometry: the theoretical predictions concern the deposition on a fibre, where the capillary pressure, set by the large curvature of the fiber, is of the same order of magnitude than the yield stress, while in the planar geometry of the plate, the capillary pressure, far smaller than the yield stress, is neglected (Maillard et al. 2014; Boujlel \& Coussot 2013). In a circular channel, the deposition of YSFs has received relatively little consideration to date, despite its relevance in numerous and diverse applications in industry (e.g. gas-assisted injection molding (Park et al. 2003), channel-cleaning processes in petroleum or food industry, displacement of hydraulic fracturing fluid (Boronin et al. 2015)) or in biomedical research (reopening of pulmonary airways (Zamankhan et al. 2012)). In this confined geometry, the capillary pressure, set by the channel's curvature typically of $10^{4} \mathrm{~m}^{-1}$, competes with the gravity and yield stresses as in the geometry of the fiber. Moreover, in a tube, the flow of YSF is characterized by an intermediate solid-liquid behaviour (Froishteter \& Vinogradov 1980): an elastic solid core appears at the center of the tube, where the shear stress is inferior to the yield stress, surrounded by a sheared liquid annulus of thickness $e$ where the shear stress overcomes the yield stress (as sketched in Figure 1):

$$
e=R-\tau_{y} L / \Delta P
$$

Where $L$ is the length of the tube and $\Delta P$ is the pressure drop between the inlet and outlet of the tube. The shape of the liquid/air interface may be altered by this solid-like region of the flow, which could modify in turn the deposition mechanism.

In this article, we report on the deposition of YSF in circular channels, and provide experimental data obtained in the limit where the capillary pressure is on the same order of magnitude than the yield stress of the fluid. Our data concern channels with various radii and over more than two orders of magnitude of flow velocities. To explain our experimental observations we develop a new scaling model that takes into account the yield stress, the capillary pressure and the viscous stress, and qualitatively describes our experimental data (for $\widehat{C a}>0.1$ ) as well as quantitatively match literature data even in the regime $\tau_{y} \lesssim T / R$, where previously published models are not applicable (for $\widehat{C a}<0.1)$.

\section{Material and Experimental set-up}

We use a Carbopol gel (980 from Cooper) as our model YSF. Carbopol is made of polyacrylic acid (PAA) resins dispersed in water and neutralized with a base. The neutralization generates ionic repulsion between the polymer chains which adopt an expanded configuration, thus forming blobs that swell until the osmotic pressure inside the blob balances the pressure inside the solvent. The size of individual blobs is of the order of $100 \mu \mathrm{m}$ (Piau 2007; Mahaut et al. 2008). Due to its non-thixotropic behaviour (Coussot et al. 2009), Carbopol gel is a good model of simple YSF.

We prepare the Carbopol solution by dispersing it in water at a concentration of 1.1 $\%$ and neutralizing the resulting solution using sodium hydroxide $(\mathrm{NaOH}, 1 \mathrm{~mol} / \mathrm{L})$. The mixing lasts 24 hours and is performed with a planetary mixer to ensure an homogeneous mixing throughout the sample. The density $(\rho)$ of the Carbopol solution we used is 980 $\mathrm{kg} / \mathrm{m}^{3}$, and its surface tension $(T)$ is $66 \mathrm{mN} / \mathrm{m}$ (Boujlel \& Coussot 2013; Jorgensen et al. 2015).

Carbopol plugs (of length $L>2 R$ ) are pushed at constant pressure in glass capillaries (radius $R=235,513,702 \mu \mathrm{m}$ ). To avoid wall slip (Barnes 1995), our glass capillaries are treated with a solution of polyethilenimine (from Sigma-Aldrich) (Metivier et al. 2012). The capillaries tube are cleaned with ethanol, dried, and finally immersed in a 

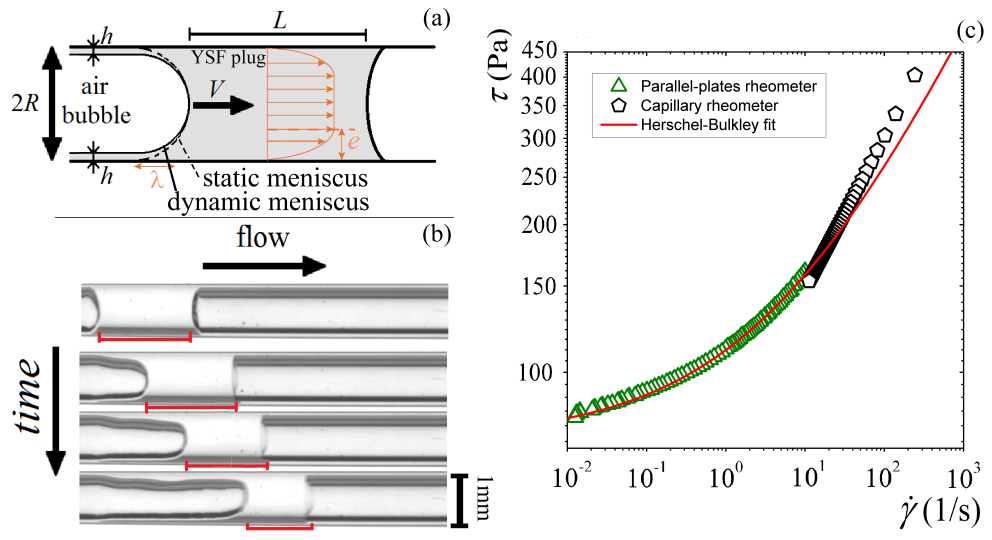

FiguRE 1. (a) Sketch of the experiment with the different lengthscales of the problem highlighted; (b) Typical images of a YSF plug as it accelerates during the channel flow for $\tau_{y}=70 \mathrm{~Pa}, R=513 \mu \mathrm{m}$, and an applied pressure $\Delta P=25 \mathrm{mbar}$. The plain lines stand for the measure of $L(t)$. The interval of time between images is equal to $0.33 \mathrm{~s}$. (c) Rheogram of the Carbobol gel used in the experiments: stress $(\tau)$ versus strain rate $(\dot{\gamma})$. Triangles are obtained with a parallel-plates rheometer, and pentagons are computed from Mooney-Rabinowitsch formula in a smooth glass capillary $(R=513 \mu \mathrm{m})$ treated with PEI to avoid wall slip. The plain line represents the Herschel-Bulkley law with $\tau_{y}=70 \mathrm{~Pa}, k=35 \mathrm{~Pa}^{n}{ }^{n}$ and $n=0.35$.

$\begin{array}{lccc}\text { Rheometer } & \tau_{y}(\mathrm{~Pa}) & k\left(\mathrm{~Pa} \cdot \mathrm{s}^{n}\right) & n \\ \text { Parallel-plates rheometer } & 74 & 38 & 0.35 \\ \text { Capillary rheometer } & 70 & 35 & 0.35\end{array}$

TABLE 1. Comparison between rheological properties of Carbopol gels measured by "classical" rheometry (rough parallel-plates rheometer) and computed from Mooney-Rabinowitsch formula in a smooth glass capillary $(R=513 \mu \mathrm{m})$ treated with PEI to avoid wall slip.

PEI solution at $0.2 \%$ w.t. in water for 24 hours. The pressure is imposed using a Fluigent pressure controller (MFCZ, 0-2 bar, \pm 2 mbar), and a movie of the experiments is recorded with a camera (AVT Marlin, maximum frame rate $200 \mathrm{fps}$ ). The maximum resolution of the camera is $2.82 \mu \mathrm{m}(1 \mathrm{px})$. Using equation 2.1 this value leads to a mean error on $h$ of approximately $20 \%$.

\subsection{Rheological properties of Carbopol gels}

Under simple shear, the rheological properties of Carbopol gels are classically modeled by an Herschel-Bulkley law: $\tau=\tau_{y}+k \dot{\gamma}^{n}$ where $\tau$ and $\dot{\gamma}$ are respectively the shear stress and the shear rate. Figure 1c shows a rheogram of a Carbopol gel over a wide range of shear rate. Low shear rates are explored with a parallel-plates rheometer (Bohlin CVOR, with rough plates of diameter $40 \mathrm{~mm}$ ) whereas large shear rates are explored using a capillary rheometer (glass capillary of radius $R=513 \mu \mathrm{m}$ ) and computed from the Mooney-Rabinowitsh formula (Macosko 1994). The measured values of $\tau_{y}, k$ and $n$ in these two configurations are reported in table 1. In the following we consider that: $\tau_{y}=70$ $\mathrm{Pa}, k=35 \mathrm{~Pa} . \mathrm{s}^{n}$ and $n=0.35$. The shear modulus of our carbopol gel is obtained by stress-strain rate measurements and found to be $100 \mathrm{~Pa}$. 
(a)

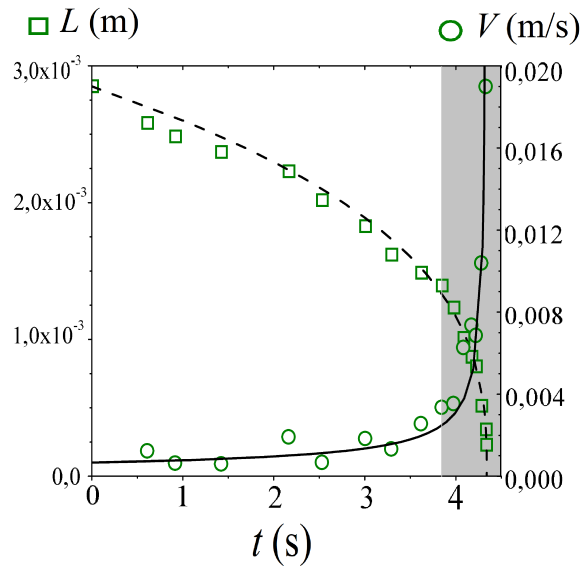

(b)

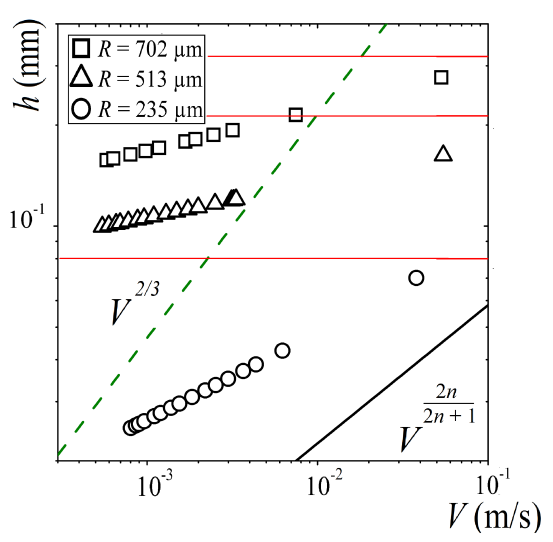

Figure 2. (a) Typical experimental data for a YSF plug pushed at constant pressure $(\Delta P=50$ mbar) in a capillary of radius $R=702 \mu \mathrm{m}$. Squares: time evolution of the length of the plug $(L)$; Circles: time evolution of the velocity of the rear meniscus of the plug $(V)$. The data points in grey area are excluded from the deposition analysis in regards to finite size effect $(L \leqslant 2 R)$. Plain and dashed lines are fitting functions used to compute equation 2.1. (b) Film thickness (h) deposited by Carbopol plugs $\left(\tau_{y}=70 \mathrm{~Pa}, k=35 \mathrm{~Pa} \cdot \mathrm{s}^{n}\right.$ and $\left.n=0.35\right)$ as a function of the bubble velocity $(V)$ in capillaries of different radii. Squares: $R=702 \mu \mathrm{m}, \Delta P=10$ mbar; triangles: $R=513 \mu \mathrm{m}, \Delta P=20$ mbar; circles: $R=235 \mu \mathrm{m}, \Delta P=30 \mathrm{mbar}$. The dashed line stands for the typical variation of the deposited thickness expected for a Newtonian fluid, and the plain line for the typical variation of the deposited thickness predicted for a shear-thinning fluid with $n=0.35$ for $R=1 \mathrm{~mm}$. The three plain red lines indicated the thicknesses of the sheared layer of YSF in the three tubes deduced from equation 1.3: $e(R=702 \mu \mathrm{m})=0.32 \mathrm{~mm}$, $e(R=513 \mu \mathrm{m})=0.21 \mathrm{~mm}$, and $e(R=235 \mu \mathrm{m})=0.08 \mathrm{~mm}$; for a given tube's radius, $h<e$.

\subsection{Measurement of the film thickness}

We deduce the film thickness $(h)$ from the length $(L)$ of YSF plugs pushed by an air bubble at constant pressure in glass capillaries. As the plug advances inside the capillary, $L$ decreases due to the deposition of the film on the capillary's wall. Its velocity $(V)$ increases in turn due to the decrease in hydrodynamic resistance associated with its flow at imposed gas pressure (Baudoin et al. 2013). This method, used to study the inertial limit of deposition of Newtonian fluids in capillaries (Aussillous \& Quéré 2000), allows to obtain a whole curve $h=f(V)$ in a single experiment. To ensure that dynamical effects are indeed negligible in YSF deposition, we also measure the layer deposited when pushing the gas at constant velocity and check that this stationary measurement coincide with the dynamical one.

The typical velocity of the experiment $(V)$, measured at the rear meniscus of the plug (i.e. the front meniscus of the bubble), typically ranges between $1 \mathrm{~mm} / \mathrm{s}$ and 20 $\mathrm{cm} / \mathrm{s}$. A sketch of the experiment, as well as consecutive images of a moving plug are shown on figure 1b. Data of $L(t)$ and $V(t)$ are presented on figure 2a. The length of the plug decreases (respectively the velocity increases) more and more rapidly during the experiment until the plug ruptures. We associate this behavior with the large viscosity contrast between the fluid and air as shown by Fujioka et al. (2008) and later on by Baudoin et al. (2013).

To avoid finite size effects (Zamankhan et al. 2012; Fujioka et al. 2008), we only consider data for which the length of the plugs $(L)$ verifies $L>2 R$, i.e. the data in dashed area of the graphs in figure $2 \mathrm{a}$ are not considered in the following. We report that when $L<2 R$ 
the film thickness tends to increase rapidly. However, due to the high speed of this regime we were not able to investigate it systematically.

Assuming that the deposit is homogeneous on the channel surface, and that the plug has a cylindrical shape, a mass balance on the moving plug yields:

$$
\frac{h}{R}=1-\sqrt{1-\frac{1}{V(t)} \frac{\mathrm{d} L}{\mathrm{~d} t}}
$$

The deposited thickness $(h)$ is thus deduced from data presented on figure 2 using equation 2.1. In equation 2.1, the film thickness is assumed to be invariant by rotation along the axis of the tube. Yet, it can be seen from figure $1 \mathrm{~b}$ that it is not always true, therefore the determined film thickness is an equivalent mean annular film thickness.

\section{Experimental results}

Data obtained using equation 2.1 for YSF plugs in capillaries of different radii are shown in a log-log plot on figure $2 \mathrm{~b}$ along with the typical power-law variation of the deposited thickness with the velocity predicted by equation 1.1 and 1.2.

Different features are visible on figure 2b. First, at a given flow velocity, increasing the radius $R$ results in a larger deposited thickness $h$. Second, for all capillary radii, $h$ increases like $V^{x}$ with $x$ varying between 0.1 for $R=513,702 \mu \mathrm{m}$ and 0.3 for $R=235$ $\mu \mathrm{m}$. This exponent $x$ is smaller than what is expected for a Newtonian fluid $(x=2 / 3)$. For the smallest value of $R$, it is close to $x=0.4$, the value expected for a non-Newtonian shear-thinning fluid with the same power-law index $n=0.35$ than the Herschel-Bulkley fluid we use (see figure 1.2). Moreover, as highlighted by the red horizontal lines of figure $2 \mathrm{~b}, h$ is always smaller than $e$, the sheared liquid layer deduced from Equation 1.3. This suggests that the deposition process is barely affected by the solid-core of the flow and indeed as $R$ decreases, the capillary pressure increases and overcomes the yield stress nearly everywhere in the channel. Thus, the solid core of the flow, characteristic of the YSF, tends to disappear, and the YSF behaves all the more like a shear thinning fluid than the tube radius is small.

To further compare these results to the classical deposition mechanism for Newtonian fluid, we represent the film thickness $h$ normalized by $R$ as a function of a modified capillary number $\widehat{C a}=k\left(\frac{V}{R}\right)^{n} /\left(\frac{T}{R}\right)$, which takes into account the shear-thinning/thickening part of the non-Newtonian character of the fluid and compare it on figure 3a to the data obtained by Taylor (1961) (using viscous fluids with a viscosity $\eta$ ranging between 0.03 and 0.079 Pa.s).

For $\widehat{C a}<1$, the thickness of YSF is smaller than what is expected for a Newtonian fluid. While for $\widehat{C a}>1$, it seems to tend to a value close to the Newtonian one. We also report that the data do not collapse on a single curve, which further implies that for a given value of $n$ all the dynamics of the coating process cannot be captured using $\widehat{C a}$ only. Since the yield stress of the fluid does not appear in this representation, this deviation is logically attributed to the intermediate solid-liquid behavior of the fluid, which contribution will be highlighted below.

\section{Model and Discussion}

In the following, we first review the deposition laws reported in the literature for different fluids. Then, by adapting the classical model for the deposition of a Newtonian fluid, we propose a new scaling law for the deposition of a YSF. 
(a)

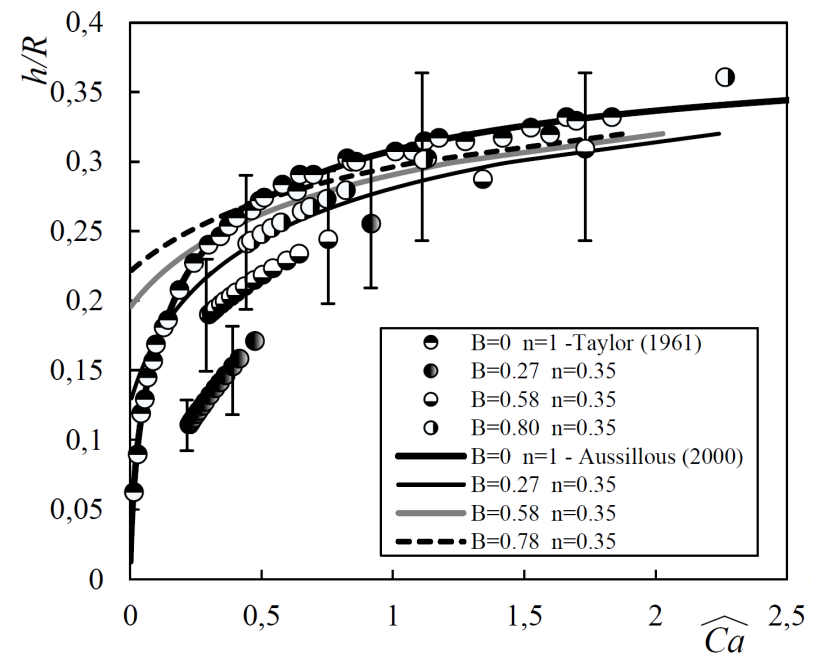

(b)

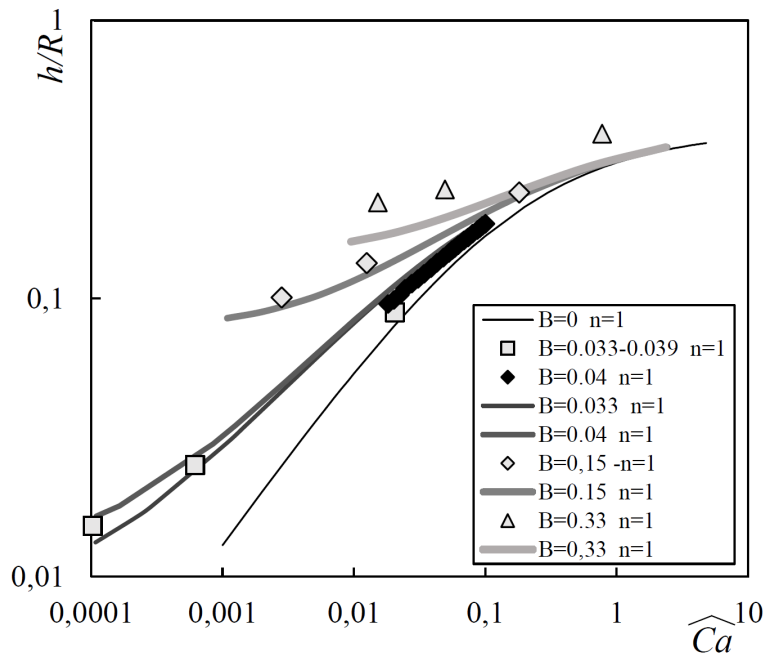

Figure 3. (a) Normalized film thickness $(h / R)$ as a function of the capillary number $\widehat{C a}$ for different capillary radii, hence different $B$. Here, $B=0.80$ corresponds to $R=702 \mu \mathrm{m}, \Delta P \simeq 10$ mbar, $\frac{e}{R}=0.46 ; B=0.58$ to $R=513 \mu \mathrm{m}, \Delta P=20 \mathrm{mbar}, \frac{e}{R}=0.42 ; B=0.27$ to $R=235$ $\mu \mathrm{m}, \Delta P=30 \mathrm{mbar}, \frac{e}{R}=0.34$ and $\Delta P=100 \mathrm{mbar}, \frac{e}{R}=0.73$. The symbols obtained by Taylor for different Newtonian fluids (Taylor 1961) are fitted by an empirical expression proposed Aussillous \& Quéré (2000) (thick black line). The three other lines (thin black, grey and dash) correspond to Equation 4.7 with $\alpha=0.33$ and $\beta=2.4$ (b) Computed values of the normalized film thickness as a function of $\widehat{C a}$ extracted from fig. 14 of the work by Zamankhan et al. (2012) (full diamonds), and from Jalaal \& Balmforth (2016) (open symbols) and fitted for their corresponding value of $B$ and $n$ using equation 4.7 and $\alpha=0.33$ and $\beta=2.4$

$$
\begin{array}{cccccc} 
& R e=\frac{\rho V^{2-n} R^{n}}{k} & B o=\frac{\rho g R^{2}}{T} & \widehat{C a}=\frac{k\left(\frac{V}{R}\right)^{n}}{T / R} & B=\frac{\tau_{y} R}{T} & B i=\frac{\tau_{y}}{k\left(\frac{V}{R}\right)^{n}} \\
\text { Range } & 10^{-5}-0.06 & 0.008-0.07 & 0.2-2.43 & 0.27-0.78 & 0.31-1.8
\end{array}
$$

TABle 2. Summary of the dimensionless number used (Re for Reynolds, Bo for Bond, Ca for capillary, Bi for Bingham) to characterize the flow and their respective ranges.

The case of a Newtonian fluid coating the inside of a circular channel was first considered by Taylor and Bretherton in conditions where gravity and inertia are negligible compared to surface tension and viscous effects (Taylor 1961; Bretherton 1961). In this limit, the deposition process can be understood as follows: the viscous stresses thicken the film in order to decrease the hydrodynamic resistance associated with the flow in the deposited layer. They also deform the meniscus with respect to its static shape in a region of length $\lambda$ where the thin film forms. The static and the deformed shape of the interface (also called the dynamic meniscus), are both represented on figure 1a) in plain and dashed lines. This change of shape generates interfacial stresses, thus leading to a gradient of capillary pressure scaling like $\frac{1}{\lambda} \frac{T}{R}$ that counteracts the viscous volumetric forces scaling like $\frac{\eta V}{h^{2}}$, with $\eta$ the fluid viscosity, $T$ its surface tension, and $V$ the velocity of the moving bubble. An analytical solution of this problem was provided by Bretherton (1961) under the lubrication approximation: in the limit where $h \ll \lambda$, matching the 
curvatures of the static and dynamic menisci provides a second equation for the length of the dynamic meniscus $\lambda$ :

$$
\lambda \sim \sqrt{h R}
$$

Combining equation 4.1 with the balance between the capillary pressure gradient and the viscous effects, the deposition law can be naturally expressed in terms of the capillary number $C a=\frac{\eta V}{T}$ leading to Equation 1.1. In the limit of relative thick film, the radius of curvature of the static meniscus is $R-h$ rather than $R$, yielding to an expression proposed by Aussillous \& Quéré (2000), which fits accurately the experimental data as can be seen in figure $3 \mathrm{a}$ :

$$
\frac{h}{R} \sim \frac{C a^{2 / 3}}{1+C a^{2 / 3}}
$$

The deposition of purely plastic materials of yield stress $\tau_{y}$ and density $\rho$ on a fibre or, respectively, on a plate, has been obtained theoretically by balancing the divergence of the stress tensor $\frac{\tau_{y}}{h}$ with the capillary pressure gradient $\frac{1}{\lambda} \frac{T}{L_{d}}$, where $L_{d}$ is the radius of the fiber or, respectively, the capillary length $\sqrt{\frac{T}{\rho g}}$. Then, using equation 4.1 (Deryagin \& Levi 1964; Quéré 1999) yields:

$$
\frac{h}{L_{d}} \sim B^{2}
$$

where $B=\frac{\tau_{y} L_{d}}{T}$ is a dimensionless number comparing the yield stress to the capillary pressure.

We emphasize the two following points concerning equation 4.3. First, equation 4.3 does not depend on flow velocity $V$, and therefore predicts a deposited film of finite thickness even in the absence of flow. Secondly, equation 4.3 has been derived using equation 4.1, which is only valid for $\frac{h}{\lambda} \ll 1$. Moreover, in terms of length of the dynamic meniscus, equation 4.3 is equivalent to $\frac{h}{\lambda} \sim B$. Thus, equation 4.3 can not be used when the yield stress is of order of the capillary pressure (or equivalently $B \simeq 1$ ).

Thus, to take into account the different contributions of the capillary, viscous and yield stresses in the limit where $B \simeq 1$, we adapt the classical models for deposition of Newtonian fluid to YSF. The dimensionless numbers used to characterize the flow and their experimental range of variation are summarized in table 2 . Since $\tau_{y}$ is quite large, there is always a competition between the yield stress and the capillary pressure jump during the flow of the Carbopol gel in our channels. To compare these two effects, we consider the dimensionless number $B=\frac{\tau_{y} R}{T}$ as suggested by Deryagin \& Levi (1964). In our experiment, $B$ is of order $1(0.27 \leqslant B \leqslant 0.78)$. Moreover, the Reynolds and Bond numbers are both $\ll 1$, thus inertial and gravity forces are negligible compared to viscous forces and surface tension (or the yield stress since $\tau_{y} \sim \frac{T}{R}$ ). Therefore, the main effects to take into account are: $(i)$ the viscous stresses of the fluid $\left(k\left(\frac{V}{h}\right)^{n}\right.$ in the dynamic meniscus); (ii) the yield stress of the fluid $\tau_{y}$; $(i i i)$ the gradient of capillary pressure between the dynamic meniscus $\left(\frac{T}{R-h}\right)$ and the static meniscus $\left(\frac{2 T}{R-\beta h}\right)$, where $\beta$ is an adjustable parameter taking into account the non-Newtonian character of the YSF. We now propose a model in the lubrication approximation to take into account our data on the full range of $\widehat{C a}$. Then, we discuss the physical ingredients of two limiting cases: $\widehat{C a} \ll 1$ and then $\widehat{C a}>1$.

Following Bretherton, we assume that lubrication approximation holds and that the flow is quasi-2D. Thus, to model the flow dynamics, we simply balance the divergence of the stress tensor and the capillary pressure gradient inside the dynamic meniscus of 
length $\lambda$. This results in equation 4.4 and its equivalent expression in dimensionless form, equation 4.5:

$$
\begin{aligned}
& \frac{\tau_{y}}{h}+\frac{k}{h}\left(\frac{V}{h}\right)^{n}=\frac{\alpha}{\lambda}\left(\frac{2 T}{R-\beta h}-\frac{T}{R-h}\right) \\
& B+\widehat{C a}\left(\frac{R}{h}\right)^{n} \sim \frac{h}{\lambda}\left(\frac{2}{1-\beta \frac{h}{R}}-\frac{1}{1-\frac{h}{R}}\right)
\end{aligned}
$$

To find the length of the dynamic meniscus $\lambda$, we match the curvature $\mathcal{C}_{\lambda}$ between the static meniscus assuming a shape of spherical cap of radius $R-\beta h$, and the dynamic meniscus of unknown curvature. In the expression above, the fitting parameter $\beta$ is used to describe an eventual thickening of the film at the bubble appex. We also report that for all moving plugs we are always able to fit the central part of the interface by a circular segment. This suggests that matching the curvature between the spherical cap and the dynamic meniscus is a suitable method to describe our results. Then, using a scaling law equivalent to the one proposed in (Aussillous \& Quéré 2000) gives:

$$
\frac{1}{R-h}+\mathcal{C}_{\lambda} \sim \frac{2}{R-\beta h}
$$

Using Equations 4.5 and 4.6 with $\mathcal{C}_{\lambda} \sim \frac{h}{\lambda^{2}}$, we compute $h, \lambda$ as a function of $R, B$ and $\widehat{C a}$ in equation 4.7. Yet, to compare the calculation to the experimental data, we introduce a matching parameter $(\operatorname{denoted} \alpha)$. For a Newtonian fluid, this numerical value was determined analytically by Bretherton $\alpha=1.34$ (Bretherton 1961). For YSFs $\alpha$ is unknown, and its numerical computation remains to be done.

$$
\widehat{C a}=\alpha\left(\frac{h}{R}\right)^{n+1 / 2}\left(\frac{2}{1-\beta \frac{h}{R}}-\frac{1}{1-\frac{h}{R}}\right)^{3 / 2}-B\left(\frac{h}{R}\right)^{n}
$$

We now discuss the different limits of equation 4.7 .

In the limit of $\widehat{C a} \ll 1$ and considering the flow of a Newtonian fluid $(B=0$ and $n=1$ ), equation 4.7 provides that $\frac{h}{R} \ll 1$ and reduces to equation 1.1 as expected. Then, for a flow of YSF in the limit of vanishing velocity, equation 4.7 reduces to 4.3 , which is consistent with the predictions made in the geometry of a fibre when gravity is negligible (Deryagin \& Levi 1964; Quéré 1999). In the confined geometry of the tube, this can be understood as follows: in the flow ahead of the bubble, the order of magnitude of the shear rate $\dot{\gamma}$ is $\frac{V}{R}$, thus in the limit of $\widehat{C a} \ll 1$, i.e. $k\left(\frac{V}{R}\right)^{n} \ll \frac{T}{R}$. Since $\frac{T}{R} \sim \tau_{y}, \tau$, the total shear stress given by $\tau=\tau_{y}+k \dot{\gamma}^{n}$, is simply $\tau \sim \tau_{y}$. Therefore, the deposited thickness depends mostly on a balance between the yield stress $\left(\tau_{y}\right)$ and the capillary pressure $\left(\frac{T}{R}\right)$, i.e. of the dimensionless number $B=\frac{\tau_{y} R}{T}$ in agreement with equation 4.3.

In the limit of $\widehat{C a}>1$, since $B \sim 1$ we expect $B i=\frac{B}{\widehat{C a}} \ll 1$ : the velocity profile along the channel section is Poiseuille-like and the total shear stress $\tau \sim k\left(\frac{V}{R}\right)^{n}$. The deposited thickness is thus expected to saturate towards a constant value as experimentally observed for Newtonian fluids in figure 3a (Taylor 1961) and shear-thinning fluids (Boehm et al. 2011).

Equation 4.7 also allows to qualitatively determine how the non-newtonian features of the constitutive equation of the Herschel-Bulkley fluid, i.e. $\tau_{y} \neq 0$ and $n \neq 1$, influence the deposition process. For a given $n$ and $h / R$, the larger $B$, the smaller $\widehat{C a}$. Thus, for a given value of $n$ the deposited thickness increases with the yield stress. This is valid whatever 
the value of $n$ : for a given $\widehat{C a}$, the thickness deposited behind a Bingham fluid is always larger than deposited behind a Newtonian fluid as shown numerically (Zamankhan et al. 2012). We also find that for shear-thinning/thickening fluid (i.e. when $\tau_{y}=0$ and $n \neq 1$ ), $h / R$ is an increasing function of $n$. The thickness deposited behind a shear-thinning fluid $(n<1)$ is smaller than deposited behind a Newtonian fluid $(n=1)$, itself smaller than deposited behind a shear-thickening fluid $(n>1)$ as previously reported in various works (Gutfinger \& Tallmadge 1965; de Ryck \& Quéré 1998; Hewson et al. 2009). However, the thickness deposited behind a Herschel-Bulkley with $\tau_{y} \neq 0$ and $n<1$ or behind a newtonian fluid $\tau_{y}=0$ and $n=1$ is less straightforward to compare. Equation 4.4 suggests that the yield stress will balance the shear-thinning effects when $\tau_{y} \sim k\left(\frac{V}{h}\right)^{n}$, i.e. when $B \sim \widehat{C a}\left(\frac{R}{h}\right)^{n}$, which equivalently writes $B / \widehat{C a}\left(\frac{h}{R}\right)^{n} \sim 1$ or $B i\left(\frac{h}{R}\right)^{n} \sim 1$. Thus, when $B i\left(\frac{h}{R}\right)^{n}>1$, the yield stress is dominant and the deposited thickness $h / R$ behind a Herschel-Bulkley fluid is larger than behind a Newtonian one, while when $B i\left(\frac{h}{R}\right)^{n}<1$, the shear thinning behavior dominates and the deposited thickness is smaller than for Newtonian fluid. This is in agreement with our experiments which are obtained for large value of $\widehat{C a}$ for which the shear-thinning behavior of the fluid is dominant.

We now move to the quantitative comparison of 4.7 and numerical results of the literature. Using a regularized constitutive equation, Zamankhan et al. (2012) studied the creeping motion of YSF liquid plugs in 2D channels and computed the evolution of $h / R$ as a function of $\widehat{C a}$ for Bingham fluids $(n=1)$ and $B=0.04$. Jalaal \& Balmforth (2016) consider Herschel-Bulkley fluid with $B$ in the range of [0.033-0.33]. Using lubrication theory, they calculate and compute $h / R$ up to value of $\widehat{C a}$ in the order of 1 by retaining the full surface curvature of the interface, which induces non-linear effects (Jalaal \& Balmforth 2016). In figure 3b, we fit the numerical data of Zamankhan et al. (2012) and Jalaal \& Balmforth (2016) (chosen for their wide range of $\widehat{C a}$ ) using equation 4.7. By adjusting the fitting parameters of equation 4.7 to their data, which gives $\alpha=0.33$ and $\beta=2.4$, we obtain an excellent agreement as long as $\widehat{C a}<0.01$. For larger value of $\widehat{C a}$, our expression underestimates the computed values of $h / R$. We also note that, while the work of Zamankhan et al. (2012) consider a parallel plate geometry, it is in good agreement with the work of Jalaal \& Balmforth (2016), and the present analytic expression, which are both derived for axisymmetric geometries. This may come from the approximation of an almost two dimensional flow in the matching region under conditions of sufficiently small values of $C a$ (Bretherton 1961; Jalaal \& Balmforth 2016). Using the same values $\alpha=0.33$ and $\beta=2.4$, we compare equation 4.7 with our experimental data in Figure 3a). Here also, we fail to describe the data, which is presumably due to the large values of $\widehat{C a}$ experienced here.

We also focus on the regime independent of the velocity $(V \rightarrow 0)$. In this regime, $h$ is supposed to depend only on the ratio of the yield stress to the capillary pressure whatever the geometry (plate, fibre, channel) as highlighted by equation 4.3 and 4.7 . Thus, in figure 4, we compare equation 4.7 in the limit of $V \rightarrow 0$ to the normalized value of the first experimentally measurable value of $h$ for $(i)$ Carbopol gels $\left(\tau_{y}=70 \mathrm{~Pa}\right)$ in tubes $(235$ $\mu \mathrm{m}<R<702 \mu \mathrm{m})$ (our data) (ii) Suspensions $\left(0.2 \mathrm{~Pa}<\tau_{y}<1.5 \mathrm{~Pa}\right)$ in tubes $(R=$ $1.93 \mathrm{~mm}$ ) (Park et al. 2003) and (iii) Carbopol gels (8 Pa $<\tau_{y}<82 \mathrm{~Pa}$ ) on plates using $\sqrt{\frac{T}{\rho g}}$ as characteristic length (Maillard et al. 2014) as a function of $B$. All data collapse on a single curve that saturates toward $h / L_{d} \sim 0.07$ when $B \rightarrow 0$, increases like $B^{2}$ for $0.3<B<1$ (as given by equation 4.3) and saturates again toward 1 for $B>1$. Since, (i) the experimental data of this study are not always obtained for completely negligible values of the viscous stress (i.e. $\widehat{C a}>0$, which is difficult to achieve experimentally due to 


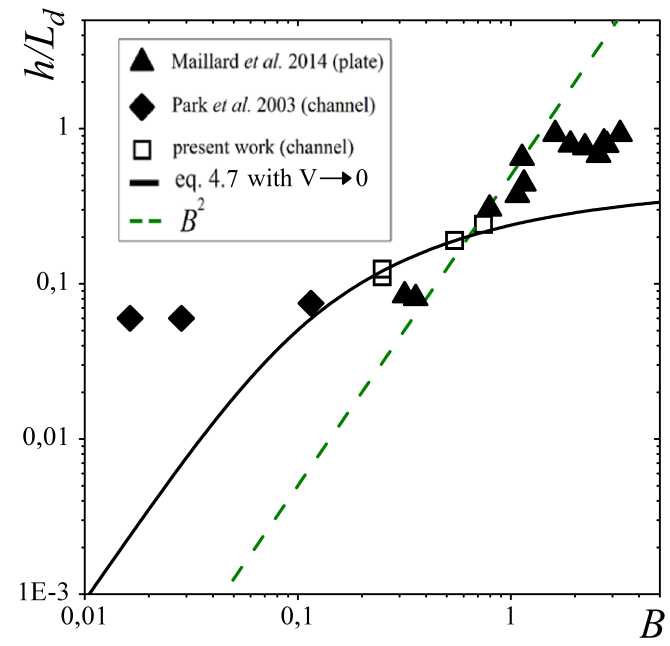

FiguRE 4. Normalized film thickness as a function of $B=\frac{\tau_{y} L_{d}}{T}$ measured for the smallest accessible velocity for literature results and our experiments: capillaries with $L_{d}=R=235$, 513, $702 \mu \mathrm{m}$ and $\tau_{y}=70 \mathrm{~Pa}$ (squares - present work ); capillaries with $L_{d}=R=1.93 \mathrm{~mm}$ and $0.2 \leqslant \tau_{y} \leqslant 1.5 \mathrm{~Pa}$ (diamonds - Park et al. (2003)); withdrawal of plate with $L_{d}=\sqrt{\frac{T}{\rho g}}$ and $8 \leqslant \tau_{y} \leqslant 82 \mathrm{~Pa}$ (triangles - Maillard et al. (2014)). The green dashed line is a guide for the eye indicating the typical $B^{2}$ variation, as in equation 4.3. The black line stands for equation 4.7 in the limit of $V \rightarrow 0$ with $\alpha=0.33$ and $\beta=2.4$.

the minimum force necessary to overcome the yield stress); (ii) as underlined previously, the lack of non-linear terms in the expression of the matching of curvature introducing errors on $h$ when either $B$ and/or $\widehat{C a}$ approach 1 , it is difficult to conclude regarding the apparent good quantitative agreement of the model and the experimental data of this study for $1<B<0.1$. However, for $B<0.1$, the measured values are significantly larger than the prediction: yield-stress fluids possess a microstructure of finite size which may set a minimal value for $h$ when $V \rightarrow 0$. Such finite size effects were reported to modify the deposition of dilute emulsions leading to a saturation of the deposited thickness (Quéré \& de Ryck 1998).

\section{Conclusion}

When there is a competition between the yield stress and the capillary pressure, for a given value of the power law index $(n)$, the deposition of a YSF in a tube cannot be described by the modified capillary number $(\widehat{C a})$ only. In the limit of vanishing $\widehat{C a}$, the yield stress enhances the deposition (quantified by $B$ ), similarly to what is observed in unconfined geometry (Deryagin \& Levi 1964; Maillard et al. 2014). Indeed, when the yield stress compete with the capillary pressure, the solid-like nature of the material imposes the deposition of a YSF layer of finite size as the velocity vanishes. The physical process of the deposition with no-slip boundary conditions, when inertial and gravity effects are negligible, is well described by a balance between the internal stresses (yield stress and viscous stress) of the fluid and the capillary pressure gradient. The model we propose is able to predict the initial deposited thickness of numerical data of literature until the film becomes too thick. For $\frac{h}{R}>0.05$, the expression we use to evaluate the curvature and the lubrication approximation do not hold anymore. We also underline that an effect of the microstructure of the fluid is expected at low velocity and that, as 
a consequence, unifying the results obtained with different materials and geometries is a challenge that requires a better understanding of the role of the microstructure and of the various effects (gravity, yield stress, capillary pressure, viscous stress, roughness of the tube).

We thank M. Maillard, I. Maimouni and J. Goyon for experimental advices. We acknowledge P. Coussot, O. Pitois and F. Gallaire for fruitful discussions. This work has benefited from two French government grants managed by ANR within the frames of the national program Investments for the Future (ANR-11-LABX-022-01) and of the young researcher program (ANR-11-JS09-012-WOLF).

\section{REFERENCES}

Ashmore, J., Shen, A. Q., Kavehpour, H. P., Stone, H. A. \& McKinley, G. H. 2008 Coating flows of non-newtonian fluids: weakly and strongly elastic limits. J. Eng. Math. 60, $17-41$.

Aussillous, P. \& QuÉRÉ, D. 2000 Quick deposition of a fluid on the wall of a tube. Phys. Fluids 12, 2367-2371.

BARnes, H. A. 1995 A review of the slip (wall depletion) of polymer solutions, emulsions and particle suspensions in viscometers: its cause, character, and cure. J. NN Fluid Mech. 56, $221-251$.

Baudoin, M., Song, Y., Manneville, P. \& Baroud, C. N. 2013 Airway reopening through catastrophic events in a hierarchical network. Proc. Natl. Acad. Sci. USA 110 (3), 859864.

Behr, M., Arora, D., Coronado, 0.M. \& M., Pasquali 2005 Gls-type finite element methods for visco-elastic fluid flow simulations. Proceedings of the third MIT Conference on Computational Fluid and Solid Mechanics pp. 586-589.

Boenm, M. W., SArker, S. \& Koelling, K. 2011 An experimental investigation of twophase coating flow within microchannels: the effect of coating fluid rheology. Microfluid. Nanofluidics 10 (6), 1175-1183.

Boronin, S. A., Osiptsov, A. A. \& Desroches, J. 2015 Displacement of yield-stress fluids in a fracture. Int. J. Multiphas. Flow 76, $47-63$.

Boujlel, J. \& Coussot, P. 2013 Measuring the surface tension of yield-stress fluids. Soft Matter 9, 5898-5908.

Bretherton, F. P. 1961 The motion of long bubbles in tubes. J. Fluid Mech. 10, 166-188.

Coussot, P., L., Tocquer, C., Lanos \& G., Ovarlez 2009 Macroscopic vs. local rheology of yield stress fluids. J. NN Fluid Mech. 158, $85-90$.

Cox, BG 1962 On driving a viscous fluid out of a tube. J. Fluid Mech. 14, 81-96.

Deryagin, B. V. \& Levi, S. M. 1964 Film Coating Theory. The Focal Press.

Duggal, R \& PASquali, M 2004 Visualization of individual DNA molecules in a small-scale coating flow. J. Rheology 48, 745-764.

Froishteter, G.B. \& Vinogradov, G.V. 1980 The laminar flow of plastic disperse systems in circular tubes. Rheologica Acta 19 (2), 239-250.

Fujioka, Hideki, Takayama, Shuichi \& Grotberg, James B. 2008 Unsteady propagation of a liquid plug in a liquid-lined straight tube. Phys. Fluids (6).

Gauri, V \& Koelling, KW 1999 The motion of long bubbles through viscoelastic fluids in capillary tubes. Rheologica Acta 38, 458-470.

Gutfinger, C. \& TAllmadge, J. A. 1965 Films of non-newtonian fluids adhering to flat plates. AIChE Journal 11 (3), 403-413.

Hewson, R. W., Kapur, N. \& Gaskell, P. H. 2009 A model for film-forming with Newtonian and shear-thinning fluids. J. N.N. Fluid Mech. 162, 21-28.

Huzyak, PC \& Koelling, KW 1997 The penetration of a long bubble through a viscoelastic fluid in a tube. J. N.N. Fluid Mech. 71, 73-88.

JalaAl, M. \& Balmforth, N.J. 2016 Long bubbles in tubes filled with viscoplastic fluid. Journal of Non-Newtonian Fluid Mechanics pp. -. 
Jorgensen, L., Le Merrer, M., Delanoe-Ayari, H. \& Barentin, C. 2015 Yield stress and elasticity influence on surface tension measurements. Soft Matter 11, 5111-5121.

KAmişLI, F. 2003 Free coating of a non-newtonian liquid onto walls of a vertical and inclined tube. Chem. Eng. Process. 42 (7), 569-581.

KAMISLI, F \& RYAn, ME 1999 Perturbation method in gas-assisted power-law fluid displacement in a circular tube and a rectangular channel. Chem. Eng. J. 75, 167-176.

KAMisLi, F \& RYAN, ME 2001 Gas-assisted non-Newtonian fluid displacement in circular tubes and noncircular channels. Chem. Eng. Sci. 56, 4913-4928.

LANDAu, L. LW \& Levich, B. 1942 Dragging of a liquid by a moving plate. ACTA Physicochimica URSS 17, 42-54.

Macosko, C. W. 1994 Rheology: principles, measurements, and applications. New York: Wiley$\mathrm{VCH}$.

Mahaut, F., Chateau, X., Coussot, P. \& Ovarlez, G. 2008 Yield stress and elastic modulus of suspensions of noncolloidal particles in yield stress fluids. J. Rheol. $\mathbf{5 2}$.

Maillard, M., Boujlel, J. \& Coussot, P. 2014 Solid-solid transition in landau-levich flow with soft-jammed systems. Phys. Rev. Lett. 112, 068304.

Metivier, C., Rharbi, Y., Magnin, A. \& Bou Abboud, A. 2012 Stick-slip control of the carbopol microgels on polymethyl methacrylate transparent smooth walls. Soft Matter $\mathbf{8}$, 7365-7367.

Park, C.-S., Baek, S.-Y., Lee, K.-J. \& Kim, S. W. 2003 Two-phase flow in a gas-injected capillary tube. Adv. Polym. Tech. 22 (4), 320-328.

Piau, J. M. 2007 Carbopol gels: Elastoviscoplastic and slippery glasses made of individual swollen sponges: Meso- and macroscopic properties, constitutive equations and scaling laws. J. NN Fluid Mech. 144, $1-29$.

Quéré, D. 1999 Fluid coating on a fiber. Annu. Rev. Fluid Mech. 31, 347-384.

QuéRÉ, D. \& DE RYCK, A. 1998 Le mouillage dynamique des fibres. In Annales de physique, , vol. 23 , pp. 1-149.

Quintella, E. F., Mendes, P. R. Souza \& Carvalho, M. S. 2007 Displacement flows of dilute polymer solutions in capillaries. J. N.N. Fluid Mech. 147, 117-128.

Reinelt, D. A. \& Saffman, P. G. 1985 The penetration of a finger into a viscous fluid in a channel and tube. SIAM J. Sci. Comput. 6 (3), 542-561.

DE RYCK, A. \& QuÉRÉ, D. 1998 Fluid coating from a polymer solution. Langmuir 14 (7), 1911-1914.

Schwartz, LW, Princen, HM \& Kiss, AD 1986 On the motion of long bubbles in capillary tubes. J. Fluid Mech. 172, 259-275.

TAYlor, G. I. 1961 Deposition of a viscous fluid on the wall of a tube. J. Fluid Mech. 10, 161-165.

Weinstein, S. J. \& Ruschak, K. J. 2004 Coating flows. Annu. Rev. Fluid Mech. 36, 29-53.

Zamankhan, P., Helenbrook, B. T., Takayama, S. \& Grotberg, J. B. 2012 Steady motion of bingham liquid plugs in two-dimensional channels. J. Fluid Mech. 705, 258-279. 